\title{
Spectroscopy of Rydberg Atoms in Non-Neutral Cold Plasmas
}

\author{
D. Feldbaum, N. V. Morrow, S. K. Dutta, G. Raithel \\ Department of Physics, University of Michigan \\ Ann Arbor, MI 48109-1120
}

\begin{abstract}
The electric field in mm-sized one-component non-neutral plasmas is measured using the Stark effect of Rydberg atoms embedded in them. The plasmas are clouds of cold $\mathrm{Rb}^{+}$-ions, which are produced by UV photoionization of laser-cooled $\mathrm{Rb}$ atoms in a magneto-optic trap. The dependence of the electric field on the number of ions and the Coulomb explosion of the ion clouds have been studied.
\end{abstract}

\section{INTRODUCTION}

Highly excited Rydberg atoms [1] are very sensitive to static and AC electric and magnetic fields, and can be used for the spectroscopic detection and compensation of such fields. Rydberg atoms have been used to detect microwave and FIR radiation [2], to measure and to compensate weak static electric fields [3], and to study QED level shifts and cavity-QED effects involving only few microwave photons [4]. The spectroscopy of Rydberg atoms has been employed to diagnose electric fields in DC and high-frequency discharge plasmas [5]. In this report, we use Rydberg atom spectroscopy to determine the electric field in millimeter-sized, strongly coupled plasmas that since recently can be generated by the laser excitation of laser-cooled clouds of atoms [6]. The field-measurement method described in this report will have future applications in the characterization of these plasmas and the study of their evolution.

\section{EXPERIMENT}

Our experiment has recently been described in Ref. [7]. In a two-step optical excitation, laser-cooled rubidium atoms confined in a volume of order $1 \mathrm{~mm}^{3}$ are first excited from the ground state $5 \mathrm{~S}_{1 / 2}$ to the $5 \mathrm{P}_{3 / 2}$ state using a $780 \mathrm{~nm}$ diode laser pulse of a few $\mu$ s duration. A short UV laser pulse $\left(\lambda_{U V}=355 \mathrm{~nm}\right.$, duration $<10 \mathrm{~ns}$, pulse energy up to $10 \mathrm{~mJ}$ ) fired during the diode laser pulse partially ionizes the $5 \mathrm{P}_{3 / 2}$-atoms, which have an ionization wavelength of $\lambda_{\text {ion }}=479.1 \mathrm{~nm}$. The resultant photoelectrons have $0.9 \mathrm{eV}$ initial kinetic energy and mostly escape on a time scale of a few ns, leaving behind a cloud of slow $\mathrm{Rb}^{+}$-ions. At a variable delay time after the $\mathrm{UV}$ pulse 
and while the $780 \mathrm{~nm}$-laser is still on, the remaining $5 \mathrm{P}_{3 / 2}$-atoms that are embedded in the ion plasma are excited by a blue dye laser pulse $(\lambda \approx 480 \mathrm{~nm}$, duration $<10 \mathrm{~ns}$, bandwidth $\approx 10 \mathrm{GHz}$ ). The blue pulse excites bound Rydberg states, the Stark effect of which we use to measure the electric fields of the non-neutral $\mathrm{Rb}^{+}$-plasma.

The Rydberg atoms are detected as follows. The ion plasma represents an electron trap with a depth $\mathrm{U}_{0}$ given by the ion number, the cloud size and the profile of the charge distribution [6]. In most of our experiments $\mathrm{U}_{0}<0.9 \mathrm{eV}$, in which case no or only few UV-generated photoelectrons are retained in the electron trap. The blue laser pulse, which is introduced shortly after the UV pulse and mostly excites bound Rydberg atoms, contains a small portion of ASE (amplified spontaneous emission) with $\lambda<$

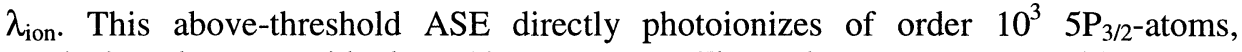
producing electrons with about $10 \mathrm{meV}$ energy. These electrons are trapped in the ion plasma. Thermal ionization of a small percentage of the bound Rydberg atoms excited by the blue pulse may add some more trapped electrons [7]. Within a few $\mu \mathrm{s}, l$ changing collisions between the trapped electrons and the Rydberg atoms efficiently promote the latter into long-lived high- $l$ states. During the Coulomb expansion of the ion plasma, the depth $\mathrm{U}_{0}$ of the plasma electron trap approaches zero. During that time, the electrons trapped in the plasma are gradually released, producing a plasma decay signal in our microchannel-plate (MCP) electron detector, which is located about $10 \mathrm{~cm}$ from the atomic cloud. The high- $l$ Rydberg atoms, which are not affected by the plasma expansion, thermally ionize with a probability of order $50 \%$ on a time scale of order $10 \mathrm{~ms}$ [7]. This time scale exceeds the plasma decay time by a factor of about $10^{4}$. The delayed thermal electrons originating in the high- $l$ Rydberg atoms are counted by the MCP detector in a gate that starts well after the plasma decay, yielding a noise-free signal of the Rydberg atom excitation. The Rydberg excitation spectra used to determine the plasma electric field are obtained by recording the delayed electron counts as a function of the wavelength of the blue laser.

Fig.1 shows a typical experimental Rydberg excitation spectrum of atoms in an electric-field-bearing plasma. Moving through the spectrum displayed in the upper panel of Fig. 1 from right to left, we observe that the electric field first manifests itself in the appearance of $p$-lines and of triangular-shaped " $h$-features", both caused by electric-field-induced state mixing. Each triangular $h$-feature reflects one manifold of hydrogenic states with the same principal quantum number $n$, which - due to the linear Stark effect - fan out over an energy range $3 n^{2} E$ (atomic units) [1]. As $n$ increases, the $h$-features progressively expand and acquire more and more overall oscillator strength. Eventually, they fill in all spectral regions of originally zero oscillator strength between the discrete non-hydrogenic $s-, p$ - and $d$-lines. The lower three panels of Fig.1 highlight the fact that there are three types of such spectral gaps, which we label $h s-$ - mid - and $p d$-gaps. The gaps become filled in exactly in that order, at quite well defined critical wavelengths. Those wavelengths can be readily converted into critical effective quantum numbers $n_{\mathrm{hs}}, n_{\text {mid }}$, and $n_{\mathrm{pd}}$. The $n_{\mathrm{i}}$ are robust indicators for the electric field, because they are solely determined by the 
general spreading behavior of the quasi-continuous $h$-features. No absolute line strength or line-strength ratio needs to be determined. Near the ionization threshold, the electric field leads to a termination of the Rydberg series at a low value of $n$ (45 in Fig. 1) and to continuum lowering.

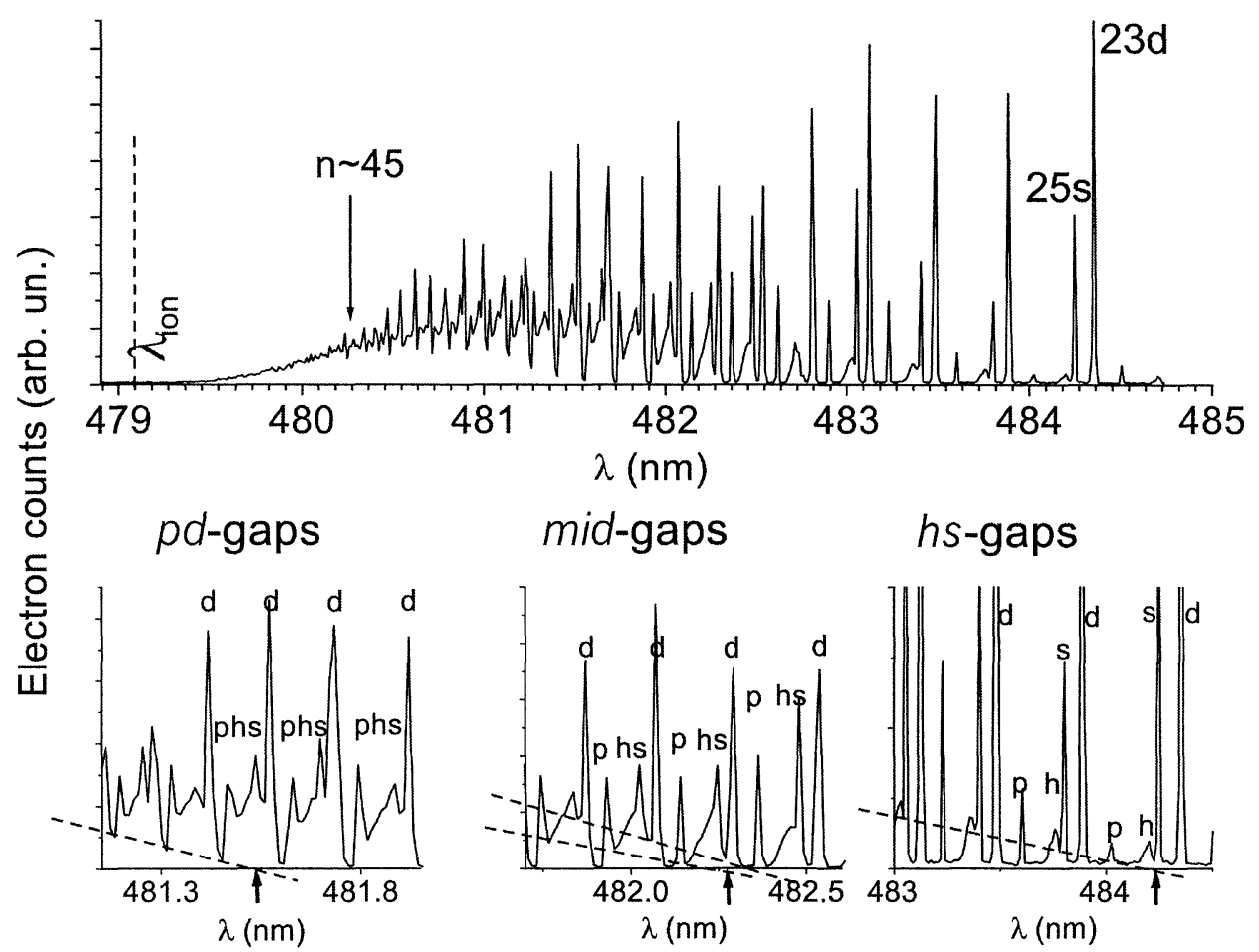

FIGURE 1 Experimental Rydberg excitation spectrum of ${ }^{87} \mathrm{Rb} 5 \mathrm{P}_{3 / 2}$-atoms embedded in a lasergenerated ion plasma. There exist three types of spectral gaps with low oscillator strength, which we denote $h s$-, mid- and $p d$-gaps. Due to the Stark effect, the spectral gaps become filled in at certain critical wavelengths that depend on the strength of the plasma electric field. The detail plots in the lower row show how we determine the critical wavelengths.

The origin of the closing of the spectral gaps becomes evident by a consideration of the Stark map of rubidium. Fig.2 shows that the $h s$-gap below the hydrogenic levels with principal quantum number $n$ disappears when the energy shift $-3 n^{2} E / 2$ of the most red-shifted hydrogenic state equals the splitting $0.13 n^{-3}$ between the unshifted hydrogenic energy $-1 /\left(2 n^{2}\right)$ and the energy of next-lower $s$-state, $-1 /\left\{2(n+0.13)^{2}\right\}$. There, the numerical value 0.13 is the non-integer part of the s-quantum defect of $\mathrm{Rb}$. The gaps between the states $(n+3) s$ and $(n+1) d$ and between the hydrogenic states $(n-1)$ and the states $(n+2) p$ close at practically identical electric fields; we label those gaps mid-gaps. Finally, the $p d$-gaps disappear when neighboring hydrogenic manifolds meet, i.e. when $3 n^{2} E=n^{-3}$. Thus, the knowledge of the quantum defects is 
sufficient to find approximate relations between electric-field strength and the above introduced critical quantum numbers $n_{\mathrm{hs}}, n_{\text {mid }}$, and $n_{\mathrm{pd}}$.

The electric field in our approximately Gaussian ion clouds follows a probability distribution, which we have calculated and found to be strongly dominated by a peak around a most probable electric field $E_{\max }$. Using a large set of isotropic excitation spectra of Rydberg atoms in fixed electric fields, and employing the electric-field probability distributions of Gaussian ion clouds as weighting functions, we have calculated model spectra of Rydberg atoms embedded in Gaussian ion clouds. The model spectra, which qualitatively appear identical to the experimental spectrum in Fig.1, have been used to establish the following relationships between the most probable electric field $E_{\max }$ and the values of $n_{\text {hs }}, n_{\text {mid }}$, and $n_{\mathrm{pd}}$ :

(field in atomic units).

$$
\begin{aligned}
& E_{\max }=0.050 n_{\mathrm{hs}}^{-5} \\
& E_{\max }=0.145 n_{\text {mid }}^{-5} \\
& E_{\max }=0.260 n_{\mathrm{pd}}^{-5}
\end{aligned}
$$

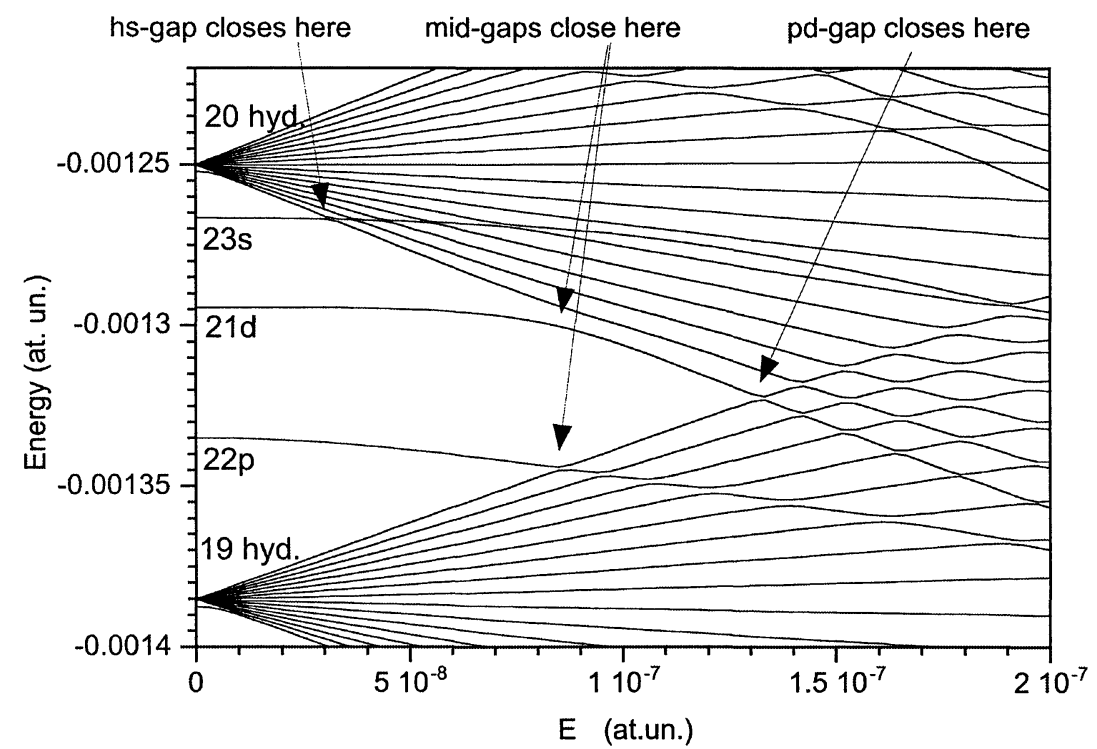

FIGURE 2 Stark map of $\mathrm{Rb}$ for magnetic quantum number $m=0$ in the vicinity of the $n=19$ and $n=20$ hydrogenic manifolds.

We have used Eqs. 1 to experimentally determine the values of $E_{\max }$ in ion clouds with diameters of $\sim 1 \mathrm{~mm}$ and various ion numbers. The delay time between the UV-pulses used to produce the ions and the blue pulses that excite Rydberg atoms embedded in the ion clouds is $23 \mathrm{~ns}$, which is long enough for the UV-generated photoelectrons to 
leave, but short enough that no significant Coulomb expansion takes place. The spectra obtained in a few such experiments are displayed in Fig.3, where a typical profile of the ion cloud at the time of its creation is also shown. The critical wavelengths $\lambda_{\text {hs }}, \lambda_{\text {mid }}$, and $\lambda_{\text {pd }}$, indicated by arrows in the right panel of Fig.3, have been determined as shown in Fig.1. Using the ionization wavelength of rubidium, $\lambda_{\text {ion }}=479.1 \mathrm{~nm}$, the corresponding critical quantum numbers $n_{\mathrm{hs}}, n_{\text {mid }}$, and $n_{\mathrm{pd}}$ are obtained from

$$
\left(\frac{1}{\lambda_{\text {ion }}}-\frac{1}{\lambda_{\mathrm{i}}}\right)=\frac{0.010974 \mathrm{~nm}^{-1}}{n_{\mathrm{i}}^{2}},
$$

where $\mathrm{i}=\mathrm{hs}$, mid or pd. Then, using Eqs.1, for each spectrum up to three spectroscopic measurements of $E_{\max }$ are obtained. The relative uncertainty of each single electricfield value is $\sim 30 \%$. Within that uncertainty, the different electric-field values obtained for the individual spectra agree well. The electric-field values quoted in Fig. 3 are the averages over all field values obtained for the respective spectra.
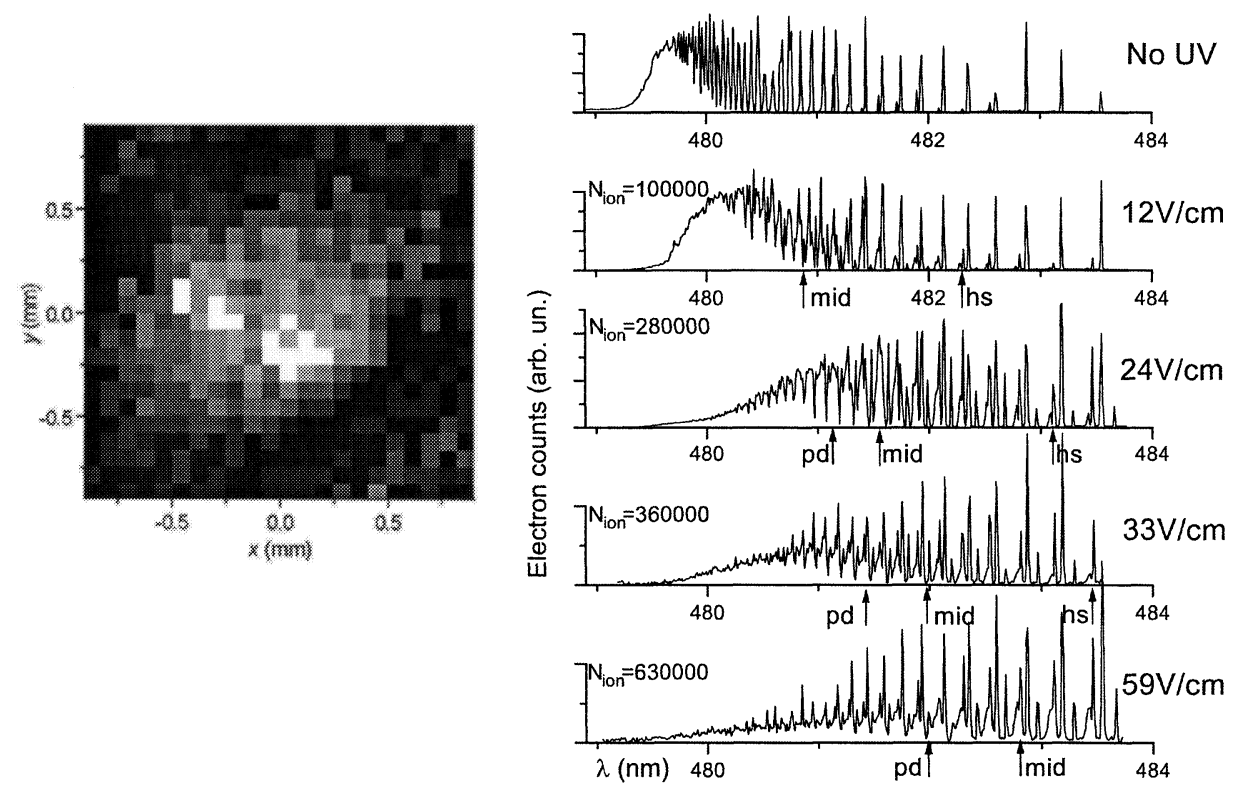

FIGURE 3

Left: Area density distribution of a typical ion cloud. The image is obtained by taking the difference between the atomic area density before and after the UV pulse. The atomic density distributions are measured by absorptive atom cloud imaging. The gray scale of the image ranges from 0 (black) to $4.5 \times 10^{7} \mathrm{~cm}^{-2}$ (white).

Right: Excitation spectra of ${ }^{87} \mathrm{Rb} 5 \mathrm{P}_{3 / 2}$-atoms embedded in mm-sized photo-excited plasmas that contain the indicated approximate numbers of $\mathrm{Rb}^{+}$-ions, $N_{\text {ion }}$. The most probable electric fields $E_{\max }$, shown on the right, are determined spectroscopically and carry an uncertainty of about $20 \%$. 


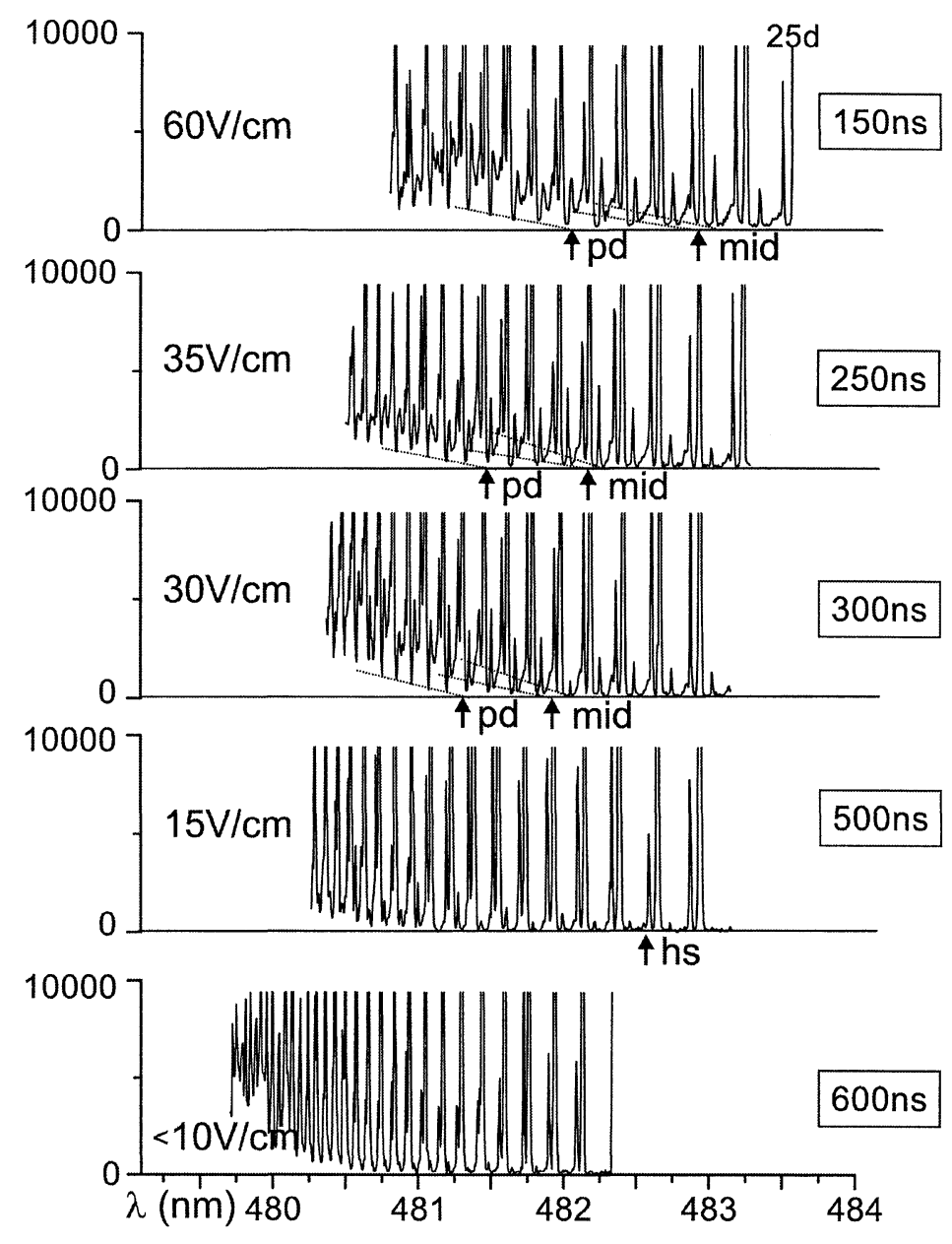

FIGURE 4 Excitation spectra of ${ }^{87} \mathrm{Rb} 5 \mathrm{P}_{3 / 2}$-atoms embedded in mm-sized photo-excited plasmas for the indicated delay times between the UV and the blue laser pulse. The most probable plasma electric fields $E_{\max }$, shown on the left, are determined from the spectra and carry an uncertainty of about $20 \%$.

Since we can vary the delay time between the UV and the blue pulse over a range of about $10 \mu \mathrm{s}$ with a precision of 10ns, the presented method of electric-field measurement is time-sensitive. Further, since the Rydberg excitation is a two-step resonant process, we can, in principle, measure the electric field vector with high spatial resolution by using crossed focused laser beams with adjustable polarizations. Temporally and spatially resolved measurements of the electric field will be an excellent tool to study the electric-field distribution, the plasma microfields (which may reveal ion ordering), and the dynamics of the plasma expansion. As a first step in that direction, we have varied the delay time between the UV and the blue pulse. A typical result, shown in Fig.4, demonstrates that the most probable electric field $E_{\max }$ 
decays over a time of order 500ns, which shows that the Coulomb explosion of the plasma cloud takes about 500ns.

\section{ACKNOWLEDGMENTS}

We acknowledge support by the NSF (Grant PHY-9875553) and partial support by the Chemical Sciences, Geosciences and Biosciences Division of the Office of Basic Energy Sciences, Office of Science, U.S. Department of Energy. Support has also been provided by the Optical Physics Interdisciplinary Laboratory (OPIL) and the FOCUS Physics Frontier Center at the University of Michigan. We further thank Prof. P. Bucksbaum for generous loaning of equipment, and B. K. Teo for assistance with the experiment.

\section{REFERENCES}

1. Gallagher, T. F., Rydberg Atoms, Cambridge University Press, Cambridge 1994.

2. Ducas, T. W., Spencer, W. P., Vaidyanathan, A. G., Hamilton, W. H., Kleppner, D., App. Phys. Lett. 35, 382 (1979), Figger, H., Straubinger, R., Walther, H., Leuchs, G., Opt. Comm. 33, 37 (1980), Gross, M., Goy, P., Fabre, C., Haroche, S., Raimond, J. M., Phys. Rev. Lett. 43, 343 (1979).

3. Neukammer, J., Rinneberg, H., Vietzke, K., Koenig, A., Hieronymus, H., Kohl, M., Grabka, H.-J., Wunner, G., Phys. Rev. Lett. 59, 2947 (1987).

4. Cavity Quantum Electrodynamics, edited by P. R. Berman, Academic Press, Inc., San Diego 1994.

5. Ganguly, B. N., J. Appl. Phys. 6, 571 (1986), Shoemaker, J. R., et al., Appl. Phys. Lett. 52, 2019 (1988), Hebner, G. A., et al., J. Appl. Phys. 76, 4036 (1994).

6. Killian, T. C., et al., Phys. Rev. Lett. 83, 4776 (1999).

7. Dutta, S. K., Feldbaum, D., Walz-Flannigan, A., Guest, J. R., Raithel, G., Phys. Rev Lett. 86, 3993 (2001). 